\title{
Importancia de cursos de prevención del suicidio en ciencias de la salud y fuerzas armadas
}

\author{
Importance of suicide prevention courses in health sciences and armed \\ forces
}

\author{
Jonatan Baños-Chaparro ${ }^{1}$ \\ Universidad Norbert Wiener
}

Recibido: $13-09-21$

Aceptado: $11-10-21$

Publicado: $23-12-21$

\section{Resumen}

Introducción: las muertes por suicidio repercuten en las familias, los miembros de la unidad y la comunidad militar. Estudios recientes señalan los diversos factores de riesgo y prevalencia de conductas suicidas en miembros de las fuerzas armadas, como también la falta de capacitación en la prevención, evaluación, intervención y posvención del suicidio en los planes de estudios de ciencias de la salud. Objetivo: describir la importancia de incluir cursos de prevención del suicidio en ciencias de la salud y fuerzas armadas. Resultados: se enfatiza la relevancia de educación sobre las conductas suicidas, los principales apartados y contenidos en estudiantes de ciencias de la salud, la formación complementaria en estudiantes y miembros de las fuerzas armadas, y los beneficios de la formación en prevención del suicidio. En ese sentido, la evidencia empírica indica que la inclusión de cursos en los planes de estudio o la capacitación adicional para profesionales de la salud y miembros de las fuerzas armadas en prevención del suicidio son favorables para la sensibilización, construcción y fortalecimiento de conocimientos sobre las conductas suicidas. Conclusiones: existe interés y motivación para garantizar que la educación esté disponible para todos, dado que una formación adecuada permite un abordaje responsable, compasivo y basado en evidencias en beneficio de la comunidad.

Palabras clave: Prevención del suicidio; educación profesional; ciencias de la salud; salud militar.

\begin{abstract}
Introduction: Suicide deaths reverberate families, unit members, and the military community. Recent studies point to the various risk factors and prevalence of suicidal behavior in members of the armed forces, as well as the lack of training in suicide prevention, evaluation, intervention, and postvention in health science curricula. Objective: describe the importance of including suicide prevention courses in health sciences and armed forces.
\end{abstract}

1 Autor para correspondencia: banos.jhc@gmail.com ORCID: https://orcid.org/0000-0002-2604-7822

(C) Los autores. Este artículo es publicado por la Revista de Investigación en Psicología de la Facultad de Psicología, Universidad Nacional Mayor de San Marcos. Este es un artículo de acceso abierto, distribuido bajo los términos de la licencia Creative Commons Atribución 4.0 Internacional (CC BY 4.0) [https://creativecommons.org/licenses/by/4.0/deed.es] que permite el uso, distribución y reproducción en cualquier medio, siempre que la obra original sea debidamente citada de su fuente original. 
Results: The relevance of education on suicidal behaviors, the main sections and contents in health sciences students, complementary training in students and members of the armed forces, and the benefits of training in suicide prevention are emphasized. In this sense, empirical evidence indicates that the inclusion of courses in the study plans or additional training for health professionals and members of the armed forces on suicide prevention are favorable for raising awareness, building and strengthening knowledge about suicide suicidal behaviors. Conclusions: There is interest and motivation to ensure that education is available to all, since adequate training allows a responsible, compassionate and evidencebased approach for the benefit of the community.

Keywords: Suicide prevention; education professional; health sciences; military health. 
Diversos profesionales de la salud y fuerzas armadas han expresado su preocupación por un probable aumento de muertes por suicidio en los últimos años. La Organización Panamericana de la Salud (OPS) informa que la quinta causa de mortalidad en la región de América es el suicidio, donde el 60\% de los casi 100 000 suicidios correspondieron a personas de 15 a 50 años de edad (OPS, 2018). Las tasas de muertes por suicidio militar en servicio activo es de 24.8/100 000 durante el 2018 en Estados Unidos (Pruitt et al., 2018), mientras que en Canada en el período del 2015-2017 la tasa de suicidios fue de 24.2/100 000 para los hombres miembros de las fuerzas armadas, donde se documentó ideación suicida previa y/o intentos de suicidio en el 38.5\% de los fallecidos (Rolland-Harris, 2018).

Las muertes por suicidio no suceden por una causa común, sino por el contrario, debido a la interacción entre una serie de factores de riesgo establecidos por la literatura científica en población general, clínica y miembros de las fuerzas armadas (Probert-Lindström et al., 2020; Ocampo-Ortega, 2020). Por ejemplo, en población general y clínica se ha identificado que los síntomas depresivos, diagnóstico de psicosis, antecedentes de intento de suicidio, problemas de consumo de sustancias y personalidad, trastorno del espectro autista, y entre otros problemas de salud mental, son indicadores de riesgo para el desarrollo de conductas suicidas (Probert-Lindström et al., 2020; Gili et al., 2019; Baños-Chaparro, 2021a; BañosChaparro, 2021b, Baños-Chaparro et al., 2021c). Del mismo modo, en las fuerzas armadas se ha evidenciado que el sexo masculino, estado civil soltero, edad mayor a 45 años, período de servicio menor a 2 años, un nivel bajo de educación, armas de fuego y diversos problemas afectivos, son factores de riesgo frecuentes en esta población (Ocampo-Ortega, 2020; Willmund et al., 2018; Baños-Chaparro, 2021d).

Una respuesta para disminuir las muertes por suicidio es establecer un plan nacional de prevención del suicidio. Estos planes están diseñados para identificar grupos de personas en probable riesgo, restringir el acceso a métodos letales, pautas para los medios de comunicación en la difusión responsable y adecuada, reducción del estigma, fortalecimiento en la atención, vigilancia y evaluación de conductas suicidas (Platt et al., 2019). Los planes nacionales reflejan el compromiso del gobierno con la población y el suicidio, siendo una apropiada respuesta para reducir las tasas nacionales. Dada su importancia, un estudio basado en 4 países europeos (Finlandia, Noruega, Suecia y Australia) que implementaron planes nacionales de prevención del suicidio, informaron reducciones considerables de muertes por suicidio a comparación de otros países que no lo hicieron (Lewitzka et al., 2019).

Las muertes por suicidio se pueden prevenir y es responsabilidad de todos poder lograrlo. Por ello, entre las estrategias de prevención del suicidio, también se encuentra como objetivo principal la educación para crear conciencia del problema en la población general, como también la capacitación de los profesionales de la salud, sectores educativos y fuerzas armadas. De esta manera, la estrategia de 
prevención del suicidio se convierte en una prioridad compartida entre diferentes profesionales al servicio de la sociedad. La falta de capacitación es un obstáculo para la prevención e intervención de conductas suicidas, dado que refuerza los mitos, creencias o actitudes acerca del suicidio, como también la evasión de discutir o preguntar sobre conductas suicidas (Ocampo-Ortega, 2020; Platt et al., 2019). Debido a este problema y las muertes por suicidio reportadas en miembros de las fuerzas armadas, población general y clínica, surge la necesidad de priorizar la formación sobre conductas suicidas en los planes de estudio de educación superior. Por lo tanto, el objetivo de esta investigación es reflexionar y describir la importancia de la educación de prevención del suicidio en los profesionales de la salud y fuerzas armadas.

\section{¿Por qué incluir la prevención del suicidio en los planes de estudio?}

Las personas que experimentan algún tipo de conducta suicida, en principio lo comunican de manera directa o indirectamente a la red de apoyo social más cercano. En esta red se incluyen los familiares, amigos y comunidad en general. Posteriormente, cuando el individuo percibe la falta de recursos para afrontar una determinada situación que le genera sufrimiento y desesperanza, acompañado de ideas suicidas, autolesiones no fatales y planificación, recurre al apoyo de un profesional de salud. Este proceso no se debe entender de manera lineal, es decir, en otras ocasiones el individuo acude directamente a un profesional de la salud sin haber solicitado el apoyo de su red social.

Un asunto importante en la búsqueda de apoyo es que no se limita estrictamente a los profesionales dedicados a la salud mental (psicólogos o psiquiatras). Por el contrario, cualquier profesional de la salud es una opción de ayuda para la persona con tendencias suicidas $\mathrm{y}$, a menudo, los profesionales de atención primaria (médicos, enfermeras, etcétera), son el puente inicial para comunicar estos comportamientos (Pruitt et al., 2018; Rolland-Harris, 2018).

Por lo tanto, a pesar que los profesionales de la salud mantienen contacto con personas que informan sobre conductas suicidas o una probabilidad de riesgo de suicidio, no cuentan con la suficiente capacitación para abordar estos casos. Un estudio reciente con participación de 67 médicos de servicios de urgencias, reveló que la mayoría no tenía los conocimientos suficientes para identificar a personas con ideas suicidas, tampoco estaban capacitados para la evaluación del riesgo de suicidio, brindar algún tipo de orientación o establecer estrategias de planes de seguridad (Alfonso et al., 2021). Mientras que una revisión sistemática concluye que los profesionales de la salud frecuentemente no reciben educación sobre el suicidio a través de sus planes de estudios (Boukouvalas et al., 2020).

Por otro lado, la Organización Mundial de la Salud (OMS) indica que, para un adecuado desarrollo de plan nacional de prevención del suicidio, un elemento 
importante es considerar la prevención desde un enfoque sostenido, coordinado y multisectorial (Platt et al., 2019). En otras palabras, la prevención no solo esta dirigida al sector de la salud pública, sino también a los sectores laborales, sociales y educativos. Las instituciones de las fuerzas armadas también son una prioridad para la prevención del suicidio. La evidencia empírica señala que los miembros de las fuerzas armadas presentan diversos problemas psicológicos, físicos y familiares, además de eventos desafiantes de la vida (por ejemplo, dificultades financieras, muerte de familiares, compañeros de trabajo o civiles) y acceso a métodos letales (armas de fuego) de forma reglamentaria o privada, siendo factores potenciales de riesgo de suicidio si no dispone de los recursos suficientes para afrontar estas situaciones de riesgo (Rolland-Harris, 2018; Baños-Chaparro, 2021d). De hecho, una revisión en población militar identificó que la prevalencia de ideación suicida de por vida se encontraba entre $13.9 \%$ y $14.1 \%$, la planificación de suicidio entre $2.3 \%$ y $5.3 \%$, mientras que el intento de suicidio entre $1.9 \%$ y $2.4 \%$ (Ocampo-Ortega, 2020).

La falta de inclusión de cursos sobre conductas suicidas en los planes de estudios, refleja la poca importancia sobre este problema de salud pública. La preocupación es aún mayor cuando el profesional de la salud atienda a una persona con conductas suicidas y no esté preparado para intervenir el problema o un miembro de las fuerzas armadas no pueda identificar o brindar un soporte adecuado en situaciones de crisis o riesgo de suicidio a otros colegas. La evidencia sugiere que la educación en los planes de estudio o la formación especializada en conductas suicidas, mejora la confianza, los conocimientos y las actitudes de los profesionales hacia el suicidio (Boukouvalas et al., 2020). En ese sentido, es fundamental que todo profesional de la salud o fuerzas armadas, tenga conocimiento básico sobre este problema de salud pública y la suficiente capacitación para la prevención, evaluación, intervención y posvención de las conductas suicidas.

\section{Cursos de prevención del suicidio en ciencias de la salud}

En principio, los estudiantes de ciencias de la salud deben comprender adecuadamente que las estrategias de prevención varían en su alcance y forma. Empezando desde la prevención universal, relacionada a brindar educación y promoción de servicios de apoyo sobre conductas suicidas en población general. También se encuentra la prevención selectiva, dirigida a grupos poblacionales vulnerables debido a diferentes situaciones de riesgo, por ejemplo: víctimas de violencia, problemas económicos, laborales o familiares, problemas psicológicos o físicos, consumo de alcohol, etcétera (Probert-Lindström et al., 2020; Gili et al., 2019; Baños-Chaparro, 2021a; Baños-Chaparro, 2021b). Por último, la prevención indicada, enfocada en grupos de individuos con manifestación de conductas suicidas o con una alta probabilidad de presentar dichas conductas dada las situaciones de crisis o factores de riesgo psicológicos, físicos y sociales (Probert-Lindström et al., 2020; Ocampo-Ortega, 2020). 
Acompañando estas aclaraciones, los estudiantes que asistan al curso de prevención del suicidio deben comprender que las clases del curso no podrán servir como su "terapia personal" de por qué un familiar o miembro de su red social decidió morir por suicidio. Por el contrario, el curso presentará la evidencia científica respecto a la prevención del suicidio, adoptando las pautas básicas de educación y capacitación sobre la identificación de factores de riesgo y protección, la evaluación y gestión de las conductas suicidas, y la intervención clínica para las personas en riesgo de suicidio.

En ese sentido, el curso de prevención del suicidio podría estar dividido en tres secciones: línea base de conocimiento, línea de intervención y línea libre (Figura 1). Por lo tanto, la línea base de conocimiento implicaría el contenido de la historia del suicidio (conceptos en el período de la antigua Grecia, período de la edad media con el surgimiento del cristianismo, período de la edad moderna, etcétera), definición sobre las conductas suicidas (ideación suicida, intento de suicidio, planificación y métodos, muerte por suicidio), aspectos epidemiológicos sobre el suicidio (prevalencia a nivel nacional y mundial), políticas de salud pública (plan nacional de prevención del suicidio) y conocimiento sobre los factores de riesgo y protección de conductas suicidas en diversas etapas de desarrollo del ser humano.

\section{Figura 1}

Secciones para el curso de prevención del suicidio en ciencias de la salud

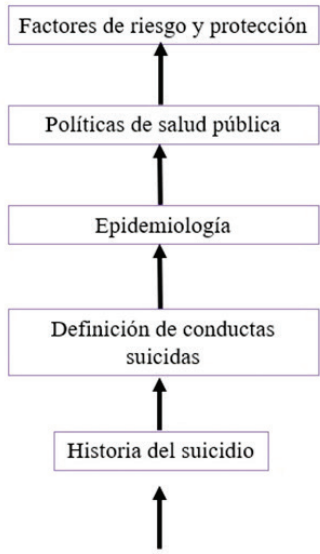

Línea base

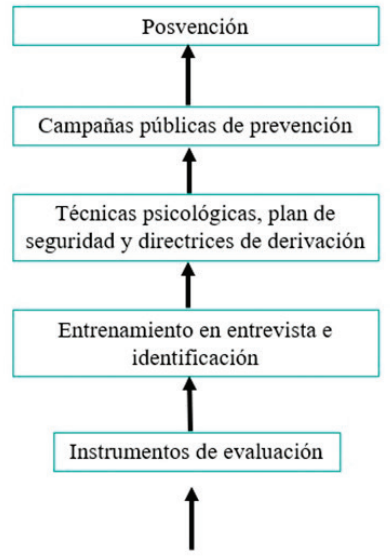

Línea intervención

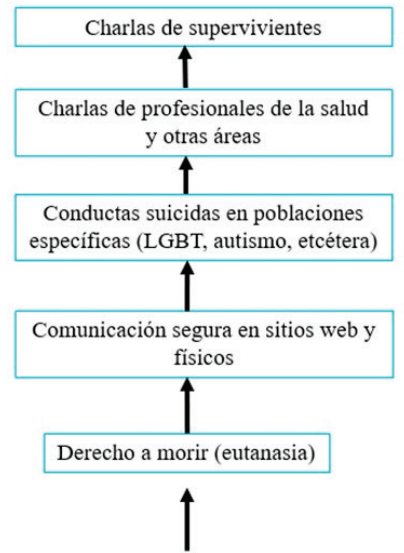

Línea libre

La línea de intervención estaría enfocada en desarrollar las competencias suficientes para un adecuado abordaje del profesional de la salud y las herramientas disponibles de apoyo que podría utilizar. Las clases estarían dirigidas a un enfoque más práctico que teórico. Por ejemplo, en la parte teórica se informa sobre los diversos instrumentos psicométricos de evaluación para las conductas suicidas, y en la parte práctica, la capacitación específica en la calificación e interpretación 
de los instrumentos de evaluación. Informar la utilidad de cada instrumento, por ejemplo: diseñado para población infantil, adolescente, adulto o condición clínica (Baños-Chaparro et al., 2021e; Baños-Chaparro \& Ramos-Vera, 2020). Asimismo, señalar la importancia de los instrumentos de cribado y extensos, aplicabilidad en entornos clínicos, escolares y población general, el objetivo de evaluación (ideación suicida pasiva o activa, autolesiones, planificación o intento de suicidio), entre otros aspectos más.

Por otro lado, las actividades también se encuentran orientadas en el entrenamiento de entrevista sobre cómo preguntar sobre conductas suicidas, identificación de factores de riesgo y protección, red de apoyo social y conversaciones complementarias con los miembros de la familia o personas más cercanas. Otra actividad importante es la intervención clínica terapéutica con técnicas psicológicas, discusiones de casos, juegos de roles, planes de seguridad y directrices de derivación a servicios de salud mental, institutos nacionales de salud especializados en la intervención de conductas suicidas, líneas telefónicas gratuitas, etcétera. Por último, esta línea de intervención podría culminar con clases acerca de las campañas públicas de prevención y la relevancia de la posvención en familiares y red social de la persona que murió por suicidio, dado que los familiares y amigos posteriormente experimentan diversos problemas psicológicos y estigma (Scocco et al., 2017).

Finalmente, la línea libre estaría dedicado a discutir sobre algunos problemas relacionados acerca del derecho a morir (eutanasia), comunicación segura en los sitios web o publicitarios físicos (carteles, folletos, etcétera), problemas de conductas suicidas en poblaciones específicas, por ejemplo: personas autoidentificadas con otra orientación sexual, personas con problemas de autismo, etcétera (BañosChaparro, 2021b; Nystedt et al., 2019). En este espacio también se podría invitar a diversos profesionales de la salud (enfermeras, médicos, psicólogos, etcétera) y profesionales de otras áreas (bomberos, asistente social, fuerzas armadas, profesionales de ciencias de la comunicación, etcétera) dedicados a la práctica profesional o investigación sobre conductas suicidas, comunicando sus actividades en función de sus puestos de trabajo. Asimismo, la participación de supervivientes (familiares de personas que murieron por suicidio) compartiendo sus experiencias personales, dificultades y redes de grupos de apoyo que existen en la comunidad.

\section{Cursos de prevención del suicidio en fuerzas armadas}

La evidencia empírica señala que los miembros de las fuerzas armadas constantemente están expuestos a diferentes situaciones de riesgo para su integridad física y psicológica. Estas situaciones se asocian mayormente con problemas de estrés, ansiedad, síntomas depresivos y diagnósticos psiquiátricos, los cuales son factores de riesgo potenciales para la emisión de conductas suicidas (Pruitt et al., 2018; Rolland-Harris, 2018; Ocampo-Ortega, 2020; Willmund et al., 2018). 
Aunque en los planes de estudio de las fuerzas armadas se encuentran dirigidas en la formación general militar y los servicios especializados, es oportuno implementar cursos electivos o talleres orientados a identificar los factores de riesgo y protección, como también los cambios de comportamientos notables (aislamiento social, reducción del interés en las actividades y tristeza) o comunicación intencional (perspectiva negativa sobre su futuro, expresión de la intención o planificación de morir, opinión acerca de la muerte o declaraciones de deseos de dejar de existir) en el personal militar (Pruitt et al., 2018; Ocampo-Ortega, 2020; Sinha et al., 2020). Una comprensión insuficiente o confusa sobre estos problemas y la poca importancia de una comunicación intencional de suicidio por parte de los familiares, amigos o líderes de la institución militar, no permitiría identificar de manera temprana los casos en probable riesgo de suicidio y derivación oportuna a los profesionales de la salud (Sinha et al., 2020).

En ese sentido, una formación complementaria en los estudiantes de las fuerzas armadas para la sensibilización de estos problemas estaría acompañada con la capacitación de la prevención del suicidio. El mensaje es claro: las muertes por suicidio son prevenibles. Por lo tanto, el contenido de los cursos electivos o talleres iniciaría con las definiciones básicas de las conductas suicidas, la identificación de factores de riesgo y protección específicamente en la vida militar, las formas de intervenir ante situaciones de riesgo o crisis (líneas telefónicas a cargo de oficiales especializados o derivación a hospitales militares del servicio de salud mental), como también limitar el acceso a método letales, por ejemplo: armas de fuego (Sinha et al., 2020). Dado que es uno de los métodos más utilizados en miembro de las fuerzas armadas, el curso tendría que ampliar más sobre este punto. Sucede que el retiro del arma podría generar vergüenza y estigma en el individuo, por lo tanto, podría considerarse algunas alternativas, por ejemplo: retirar el percutor y permitir aún la posesión de arma, guardar en lugares seguros y sin munición (caja fuerte) o que otra persona tenga la custodia, limitación temporal y orientación de seguridad (seguro de gatillo) (Baños-Chaparro, 2021d). Asimismo, brindar estrategias de comunicación y búsqueda de apoyo, campañas de prevención del suicidio (mensajes difundidos a través de vídeos, carteles y folletos para reducir el estigma de buscar ayuda) y participación de supervivientes militares (Figura 2). El objetivo del curso electivo o taller es que los estudiantes tengan conocimiento sobre este problema de salud pública que también se encuentra presente en miembros de las fuerzas armadas y, asimismo, conozcan las herramientas suficientes para afrontar situaciones de riesgo y detectar estas conductas en otros compañeros.

Por otro lado, la educación sobre la prevención del suicidio también estaría dirigida a los líderes de la institución militar de una manera más especializada y al servicio de la comunidad militar. De modo que, cuando un miembro de la unidad presente problemas de conductas suicidas, acuda al líder de la institución en búsqueda de apoyo y orientación. Las implicancias del líder capacitado tendrían 
bastantes beneficios, dado que el líder conoce las actividades y responsabilidades que conlleva la vida militar, los problemas recurrentes que atraviesan los miembros de la unidad y la derivación adecuada a los centros de salud especializados o grupos de apoyo. De hecho, actualmente se desarrollan herramientas específicas para la prevención del suicidio para líderes en el Ejército, cuyo propósito es abordar estas necesidades desde la institución militar. El estudio refiere que los líderes pueden realizar diversas actividades recomendables (Figura 2). Por ejemplo: conversaciones cara a cara con los soldados y otros miembros importantes (familiares, amigos y compañeros de la unidad) para una mejor comprensión y abordaje del problema, reuniones entre líderes para discutir sobre los soldados en probable riesgo, seguimiento de casos focalizados, reuniones mensuales multidisciplinares (batallón, brigada, divisón, pelotón, compañía) y profesionales (líderes, oficiales, capellanes) para la toma de decisiones, entre otras actividades (Curley et al., 2019). En consecuencia, tanto los estudiantes y líderes de las instituciones militares se convierten en miembros activos de la prevención del suicidio, siendo una estrategia favorable para identificar probables casos en riesgo y sensibilización para la búsqueda de ayuda profesional.

\section{Figura 2}

Secciones para el curso de prevención del suicidio en fuerzas armadas y actividades de los lideres militares
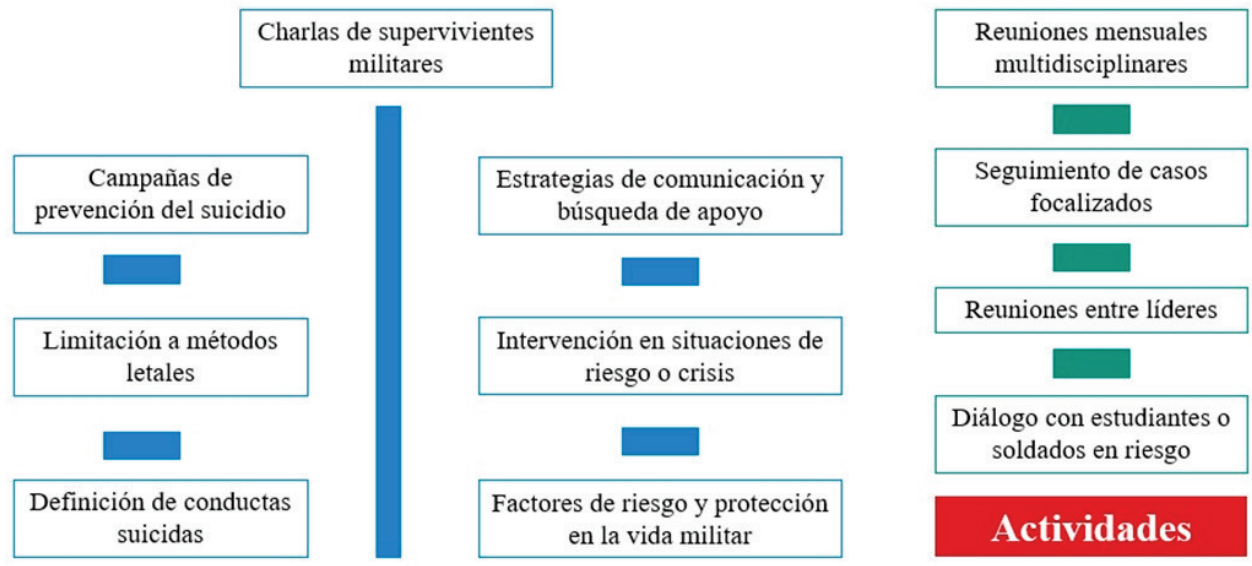

\section{Estudiantes \\ Militares}

\section{Líderes \\ Militares}

\section{Beneficios de la formación sobre prevención del suicidio}

Los profesionales de la salud, más allá de la práctica privada, también es probable que presenten servicios en otras instituciones, por ejemplo, educación, centros públicos especializados en violencia familiar y sexual, fuerzas armadas, compañías 
mineras, organizaciones no gubernamentales, entre otros. Del mismo modo, los profesionales de las fuerzas armadas en instituciones educativas militares, recursos humanos, comunicaciones, hospitales, servicios comunitarios, etcétera. En ambas disciplinas, se evidencia una amplia red de servicios que proporciona una mayor comunicación con población general y clínica, manteniendo inclusive contacto con personas en probable riesgo de conductas suicidas (Alfonso et al., 2021; OcampoOrtega, 2020; Sinha et al., 2020).

La formación de prevención del suicidio tiene múltiples beneficios para los participantes y comunidad. Un estudio longitudinal que desarrolló un curso de suicidología en pregrado de ciencias de la salud, evidenció un impacto sólido y positivo en los participantes. Asimismo, experimentaron grandes avances en el conocimiento fundamental sobre la prevención del suicidio y reducciones significativas en su estigma y actitudes negativas hacia el suicidio en comparación del grupo control (Muehlenkamp \& Thoen, 2019). Experiencias similares también se evidencian en líderes y miembros de las instituciones militares, dado que algunos miembros de las fuerzas armadas presentan algunas barreras para buscar ayuda profesional: vergüenza, desconfianza en sus superiores y preocupaciones sobre ser tratados de manera diferente (Curley et al., 2019; VanSickle et al., 2016; Wyman et al., 2020). Los cursos de prevención del suicidio pueden reducir estas barreras para solicitar apoyo. Inclusive, en estudiantes de ciencias de la salud, existe mayores probabilidades de convertirse en defensores activos de la prevención del suicidio en sus comunidades y lugares de trabajo (Muehlenkamp \& Thoen, 2019).

El contenido de la formación podría dividirse en base a criterios de prioridad. Algunos temas podrían ser consideradores apropiados en los primeros años de estudio y otros temas para los últimos años, asegurando una educación continua sobre la prevención del suicidio. Por otro lado, las investigaciones recientes sobre los factores de riesgo en población general, clínica y fuerzas armadas, brinda información relevante sobre los individuos potencialmente vulnerables (ProbertLindström et al., 2020; Ocampo-Ortega, 2020). La importancia y propuesta de cursos de prevención del suicidio en ciencias de la salud y fuerzas armadas, ofrece grandes oportunidades para ambas disciplinas. Un abordaje responsable, compasivo y basado en evidencias en beneficio de la comunidad.

\section{CONCLUSIONES}

Las muertes por suicidio repercuten en las familias, los miembros de la unidad y la comunidad militar. Estudios recientes señalan los diversos factores de riesgo y prevalencia de conductas suicidas en miembros de las fuerzas armadas, como también la falta de capacitación en la prevención, evaluación, intervención y posvención del suicidio en los planes de estudios de ciencias de la salud. Dada esta situación, surge la necesidad de incluir cursos de prevención del suicidio para intervenir conjuntamente los casos en probable riesgo y reducir el número de muertes por suicidio. 
Las estrategias nacionales para la prevención del suicidio enfatizan la educación tanto de los profesionales, estudiantes y comunidad en general. La evidencia empírica indica que los cursos, capacitaciones o talleres de prevención del suicidio resultan favorables y positivos para sensibilizar, construir y fortalecer conocimientos acerca de las conductas suicidas. Proporcionar evidencia de que la educación produce resultados positivos también puede influir en la creación de una ley nacional que considere la educación continua de la prevención del suicidio en sectores de la salud y educación. Por lo tanto, existe interés y motivación para garantizar que la educación en la prevención e intervención del suicidio esté disponible para todos.

\section{Fuentes de financiamiento}

La investigación fue autofinanciada por el propio autor.

\section{Conflicto de intereses}

El autor declara no tener ningún conflicto de interés económico, institucional, laboral o personal al realizar el manuscrito.

\section{REFERENCIAS}

Alfonso Villalba, J. M., Callizo Bedoya, M. A., Chaparro Garay, G. A., Chiola Sanabria, S. A., Dorrell Stewart, P., De la Hoz Valdez, M. L., Penner Sawatzky, D., Vargas Diez Pérez, M. A., García, O., \& Torales, J. (2021). Conocimientos y prácticas de médicos de urgencias de un Hospital Universitario sobre la atención de pacientes suicidas. Revista virtual de la Sociedad Paraguaya de Medicina Interna, 8(1), 94-105. https:// doi.org/10.18004/rvspmi/2312-3893/2021.08.01.94

Baños-Chaparro, J. (2021a). Comportamiento suicida en el trastorno del espectro autista. Revista Española de Discapacidad, 9(1), 285-294. https://doi.org/10.5569/2340$\underline{5104.09 .01 .16}$

Baños-Chaparro, J. (2021b). Síntomas depresivos en adultos peruanos: un análisis de redes. Revista virtual de la Sociedad Paraguaya de Medicina Interna, 8(2), 11-22. https://www.revistaspmi.org.py/index.php/rvspmi/article/view/243

Baños-Chaparro, J. (2021d). Suicidio por arma de fuego: un problema de salud pública. Medisur, 19(2), 342-345. http://www.medisur.sld.cu/index.php/medisur/article/ view/4973

Baños-Chaparro, J., \& Ramos-Vera, C. (2020). Validez e invarianza según sexo y edad de la Escala Paykel de Ideación Suicida en adolescentes peruanos. Interacciones, 6(1), e225. https://doi.org/10.24016/2020.v6n1.225

Baños-Chaparro, J., Ynquillay-Lima, P., Lamas Delgado, F., \& Fuster Guillen, F. G. (2021c). Análisis de redes de las conductas suicidas en adultos peruanos. Revista $\mathrm{Cu}$ bana de Medicina Militar, 50(3), e02101450. http://revmedmilitar.sld.cu/index.php/ $\mathrm{mil} /$ article/view/1450/1004 
Baños-Chaparro, J., Ynquillay-Lima, P., Lamas Delgado, F., \& Fuster Guillen, F. G. (2021e). Inventario de Frecuencia de Ideación Suicida: evidencias psicométricas en adultos peruanos. Revista Información Cientifica, 100(4), e3507. http://www.revinfcientifica.sld.cu/index.php/ric/article/view/3507

Boukouvalas, E., El-Den, S., Murphy, A. L., Salvador-Carulla, L., \& O'Reilly, C. L. (2020). Exploring Health Care Professionals' Knowledge of, Attitudes Towards, and Confidence in Caring for People at Risk of Suicide: A Systematic Review. Archives of Suicide Research, 24(2), 1-32. https://doi.org/10.1080/13811118.2019.1586608

Curley, J. M., Penix, E. A., Srinivasan, J., Sarmiento, D. M., McFarling, L. H., Newman, J. B., \& Wheeler, L. A. (2019). Development of the U.S. Army's Suicide Prevention Leadership Tool: The Behavioral Health Readiness and Suicide Risk Reduction Review (R4). Military Medicine, 185(5-6), e668-777. https:/doi.org/10.1093/milmed/ usz380

Gili, M., Castellví, P., Vives, M., Torre-Luque, A., Almenara, J., Blasco, M. J., Cebria, A. I., Gabilondo, A., Pérez-Ara, M. A., Miranda-Mendizábal, A., Lagares, C., ParésBadell, O., Piqueras, J. A., Rodríguez-Jiménez, T., Rodríguez-Marín, J., Soto-Sanz, V., Alonso, J., \& Roca, M. (2019). Mental disorders as risk factors for suicidal behavior in young people: A meta-analysis and systematic review of longitudinal studies. Journal of Affective Disorders, 245, 152-162. https://doi.org/10.1016/j.jad.2018.10.115

Lewitzka, U., Sauer, C., Bauer, M., \& Felber, W. (2019). Are national suicide prevention programs effective? A comparison of 4 verum and 4 control countries over 30 years. BMC Psychiatry, 158, 19. https://doi.org/10.1186/s12888-019-2147-y

Muehlenkamp, J. J., \& Thoen, S. K. (2019). Short- and Long-Term Impact of an Undergraduate Suicidology Course. Suicide and Life-Threatening Behaviors, 49(6), 1573 1586. https://doi.org/10.1111/sltb.12552

Nystedt, T., Rosvall, M., \& Lindström, M. (2019). Sexual orientation, suicide ideation and suicide attempt: A population-based study. Psychiatry Research, 275, 359-365. https://doi.org/10.1016/j.psychres.2019.04.007

Ocampo-Ortega, R. (2020). Riesgo de suicidio en militares. Revista Cubana de Medicina Militar, 49(1), 157-174. http://www.revmedmilitar.sld.cu/index.php/mil/article/ view/363/441

Organización Panamericana de la Salud. (2018). La carga de los trastornos mentales en la región de las Américas, 2018. DC: OPS.

Platt, S., Arensman, E., \& Rezaeian, M. (2019). National Suicide Prevention Strategies - Progress and Challenges. Crisis, 40(2), 75-82. https://doi.org.10.1027/0227-5910/ $\underline{\mathrm{a} 000587}$

Probert-Lindström, S., Berge, J., Westrin, A., Öjehagen, A., \& Skogman Pavulans, K. (2020). Long-term risk factors for suicide in suicide attempters examined at a medical emergency in patient unit: results from a 32-year follow-up study. BMJ Open, 10, e038794. https://doi.org/10.1136/bmjopen-2020-038794 
Pruitt, L. D., Smolenski, D. J., Tucker, J., Issa, F., Chodacki, J., McGraw, K., \& Kennedy, C. H. (2018). DoDSER: Department of Defense suicide event report — calendar year 2017 annual report. https://www.dspo.mil/Portals/113/Documents/2017-DoDSERAnnual-Report.pdf?ver=2019-07-19-110951-577

Rolland-Harris, E. (2018). 2018 Report on Suicide Mortality in the Canadian Armed Forces (1995 to 2017). https://www.canada.ca/en/department-national-defence/ corporate/reports-publications/health/2018-report-on-suicide-mortality-in-the-caf1995-to-2017.html

Scocco, P., Preti, A., Totaro, S., Ferrari, A., \& Toffol, E. (2017). Stigma and psychological distress in suicide survivors. Journal of Psychosomatic Research, 94, 39-46. https:// doi.org/10.1016/j.jpsychores.2016.12.016

Sinha, A., Gupta, S., Ray, M., Kumar, S., \& Kumar Gupta, A. (2020). Lessons Learned from Psychological Autopsies in Armed Forces. Indian Journal of Psychology Medicine, 43(2), 150-153. https://doi.org/10.1177/0253717620950254

VanSickle, M., Werbel, A., Perera, K., Pak, K., DeYoung, K., \& Ghahramanlou-Holloway, M. (2016). Perceived barriers to seeking mental health care among United States Marine Corps noncommissioned officers serving as gatekeepers for suicide prevention. Psychology Assessment, 28(8), 1020-1025. https://doi.org/10.1037/pas0000212

Willmund, G. D., He, J., Helms, C., Wertenauer, F., Seiffert, A., Nolte, A., Wesemann, U., \& Zimmermann, P. L. (2018). Suicides between 2010 and 2014 in the German Armed Forces - Comparison of Suicide Registry Data and a German Armed Forces Survey. Suicide and Life-Threatening Behaviors, 49(5), 1497-1509. https://doi.org/10.1111/ sltb. 12534

Wyman, P. A., Pisani, A. R., Yates, B., Morgan-DeVelder, L., Schmeelk-Cone, K., Gibbons, R. D., Caine, E. D., Petrova, M., Neal-Walden, T., Linkh, D. J., Matteson, A., Simonson, J., \& Pflanz, S. E. (2020). Effect of the Wingman-Connect Upstream Suicide Prevention Program for Air Force Personnel in Training. JAMA Netw Open, 3(10), e2022532. https://doi.org/10.1001/jamanetworkopen.2020.22532 